\title{
Synthesis and Characterization of New Phenylbis(salicylato)bismuth(III) Complexes
}

\author{
Vitalie Stavila $^{1}$, James C. Fettinger ${ }^{2}$ and Kenton H. Whitimire ${ }^{1}$. \\ ${ }^{1}$ Department of Chemistry, MS 60, Rice University, 6100, Main Street, Houston, Texas 77005-1892 \\ ${ }^{2}$ Department of Chemistry, University of California, One Shields Ave., Davis, California 95616-5298 \\ *E-mail: whitmir@ rice.edu
}

\section{SUPPORTING INFORMATION}

Figure S1. ESI-MS of (a) (1a) and (b) (1b) in negative mode.

Figure S2. Observed and calculated isotope distributions for (a) [BiPh(Hsal)(sal)] $]^{-}$and (b) $\left[\mathrm{Bi}_{2} \mathrm{Ph}_{2}(\mathrm{Hsal})(\mathrm{sal})_{2}\right]^{-}$in ESI-MS.

Figure S3. Observed and calculated isotope distributions for (a) $\left[\mathrm{BiPh}\left(\mathrm{Hsal}^{4 \mathrm{Me}}\right)\left(\mathrm{sal}^{4 \mathrm{Me}}\right)\right]^{-}$and (b) $\left[\mathrm{Bi}_{2} \mathrm{Ph}_{2}\left(\mathrm{Hsal}^{4 \mathrm{Me}}\right)\left(\mathrm{sal}^{4 \mathrm{Me}}\right)_{2}\right]^{-}$in ESI-MS.

Figure S4. ${ }^{1} \mathrm{H}$ NMR spectra of (a) $\mathbf{1 a}$ and (b) $\mathbf{1 b}$ in $\mathrm{d}_{6}$-dmso (400 MHz).

Figure S5. ${ }^{13} \mathrm{C}\left\{{ }^{1} \mathrm{H}\right\}$ NMR spectra of (a) $\mathbf{1 a}$ and (b) $\mathbf{1 b}$ in $\mathrm{d}_{6}$-dmso (400 MHz).

Figure S6. ${ }^{1} \mathrm{H}-{ }^{1} \mathrm{H}$ COSY NMR spectra of $\mathbf{2 a}$ and $\mathbf{2 c}$ in $\mathrm{d}_{6}$-dmso $(500 \mathrm{MHz})$.

Figure S7. Coordination environment of $\mathrm{Bi}(\mathrm{III})$ in the two molecules of the asymmetric unit of $\mathbf{2 c}$.

Table S1. Selected geometric parameters for $\mathbf{2 c}$.

Figure S8. Coordination environment of $\mathrm{Bi}(\mathrm{III})$ in $[3]_{2} \cdot 2 \mathrm{Me}_{2} \mathrm{CO}$ and $[4]_{2} \cdot 4 \mathrm{Me}_{2} \mathrm{CO}$.

Figure S9. The negative-ion ESI mass spectrum of the product of the reaction of $\mathrm{BiPh}_{3}$ and $\mathrm{H}_{2} \mathrm{Sal}^{4 \mathrm{Me}}$ in reagent-grade acetone.

Figure S10. Comparison of experimental and calculated negative-ion ESI mass spectral isotope patterns for $\left[\mathrm{Bi}_{9} \mathrm{O}_{7}\left(\mathrm{Hsal}^{4 \mathrm{Me}}\right)_{13}\left(\mathrm{sal}^{4 \mathrm{Me}}\right)\right]^{2-}$ and $\left[\mathrm{Bi}_{10} \mathrm{O}_{8}\left(\mathrm{Hsal}^{4 \mathrm{Me}}\right)_{14}\left(\mathrm{sal}^{4 \mathrm{Me}}\right)\right]^{2}$.

Figure S11. Comparison of experimental and calculated negative-ion ESI mass spectral isotope patterns for $\left[\mathrm{Bi}_{10} \mathrm{O}_{8}\left(\mathrm{Hsal}^{4 \mathrm{Me}}\right)_{14}\left(\mathrm{sal}^{4 \mathrm{Me}}\right)\left(\mathrm{Me}_{2} \mathrm{CO}\right)_{2}\left(\mathrm{H}_{2} \mathrm{O}\right)_{2}\right]^{2-}$ and $\left[\mathrm{Bi}_{10} \mathrm{O}_{8}\left(\mathrm{Hsal}^{4 \mathrm{Me}}\right)_{14}\left(\mathrm{sal}^{4 \mathrm{Me}}\right)\left(\mathrm{Me}_{2} \mathrm{CO}\right)_{2}\left(\mathrm{H}_{2} \mathrm{O}\right)_{5}\right]^{2-}$.

Table S2. Attenuated total reflectance FT-IR spectra for complexes 1a, 1b, 2a- 2d, 5. 

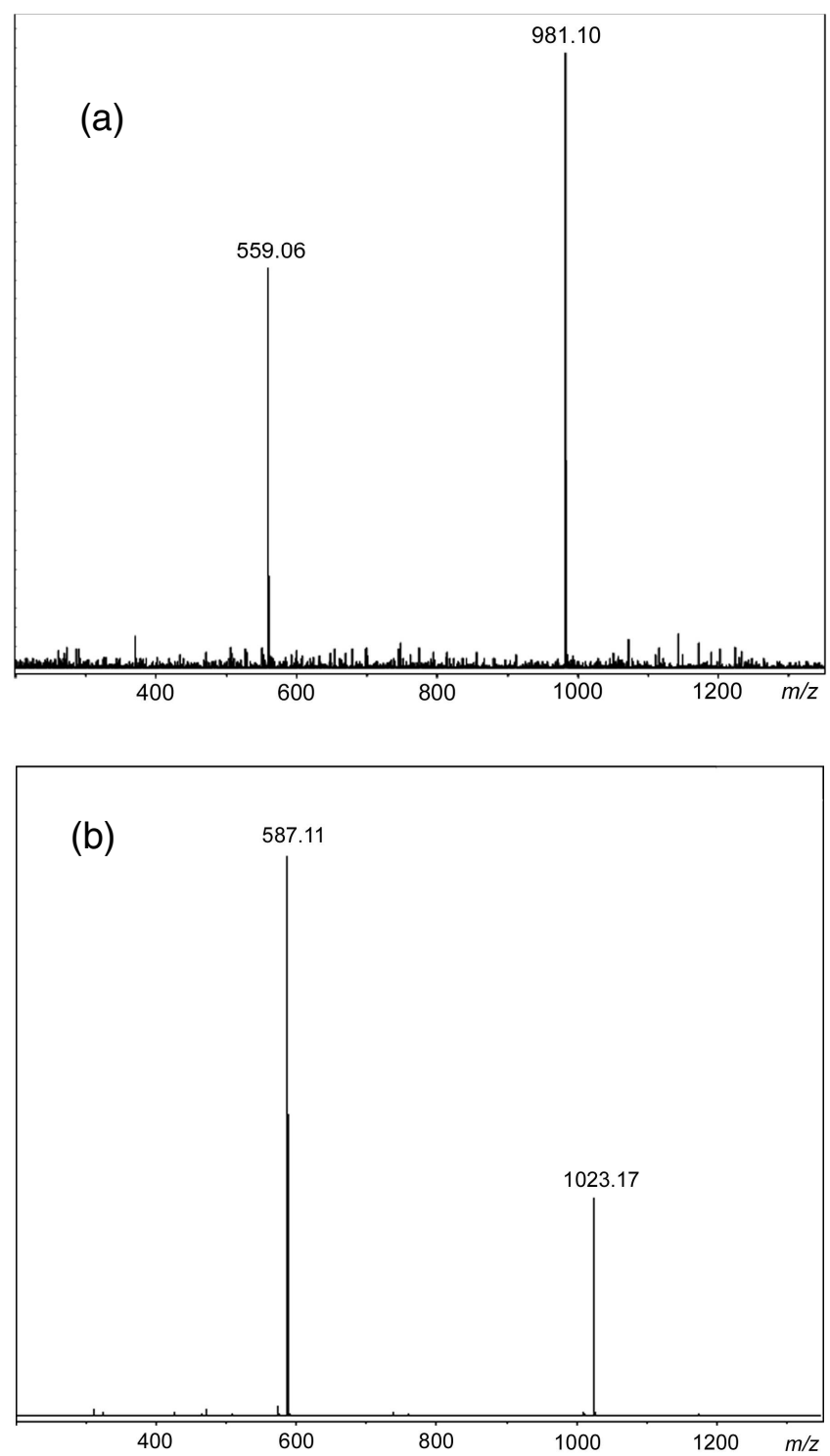

Figure S1. ESI-MS of (a) (1a) and (b) (1b) in negative mode. The two sets of prominent peaks at $\mathrm{m} / \mathrm{z}$ 559.06 and 981.10 (for 1a) and 587.11 and 1023.17 (for $\mathbf{1 b})$ correspond to $\left[\mathrm{Bi}(\mathrm{Ph})(\mathrm{Hsal} *)\left(\mathrm{sal}^{*}\right)\right]^{-}$and $\left[\mathrm{Bi}_{2}(\mathrm{Ph})_{2}\left(\mathrm{Hsal}^{*}\right)\left(\mathrm{sal}^{*}\right)_{2}\right]^{-}$ 

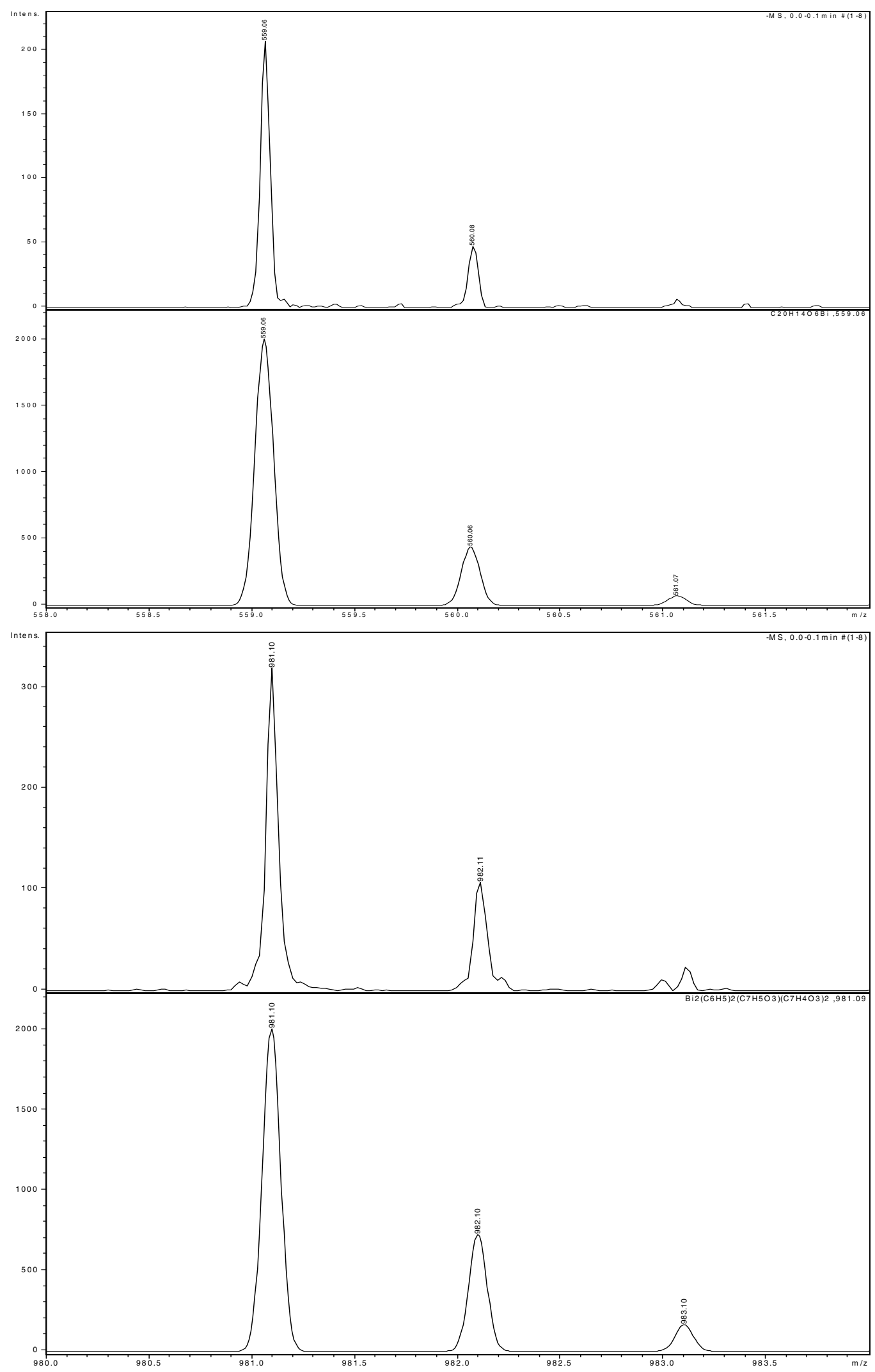

Figure S2. Observed and calculated isotope distributions for (a) $[\mathrm{BiPh}(\mathrm{Hsal})(\mathrm{sal})]^{-}$and (b) $\left[\mathrm{Bi}_{2} \mathrm{Ph}_{2}(\mathrm{Hsal})(\mathrm{sal})_{2}\right]^{-}$in ESI-MS. 

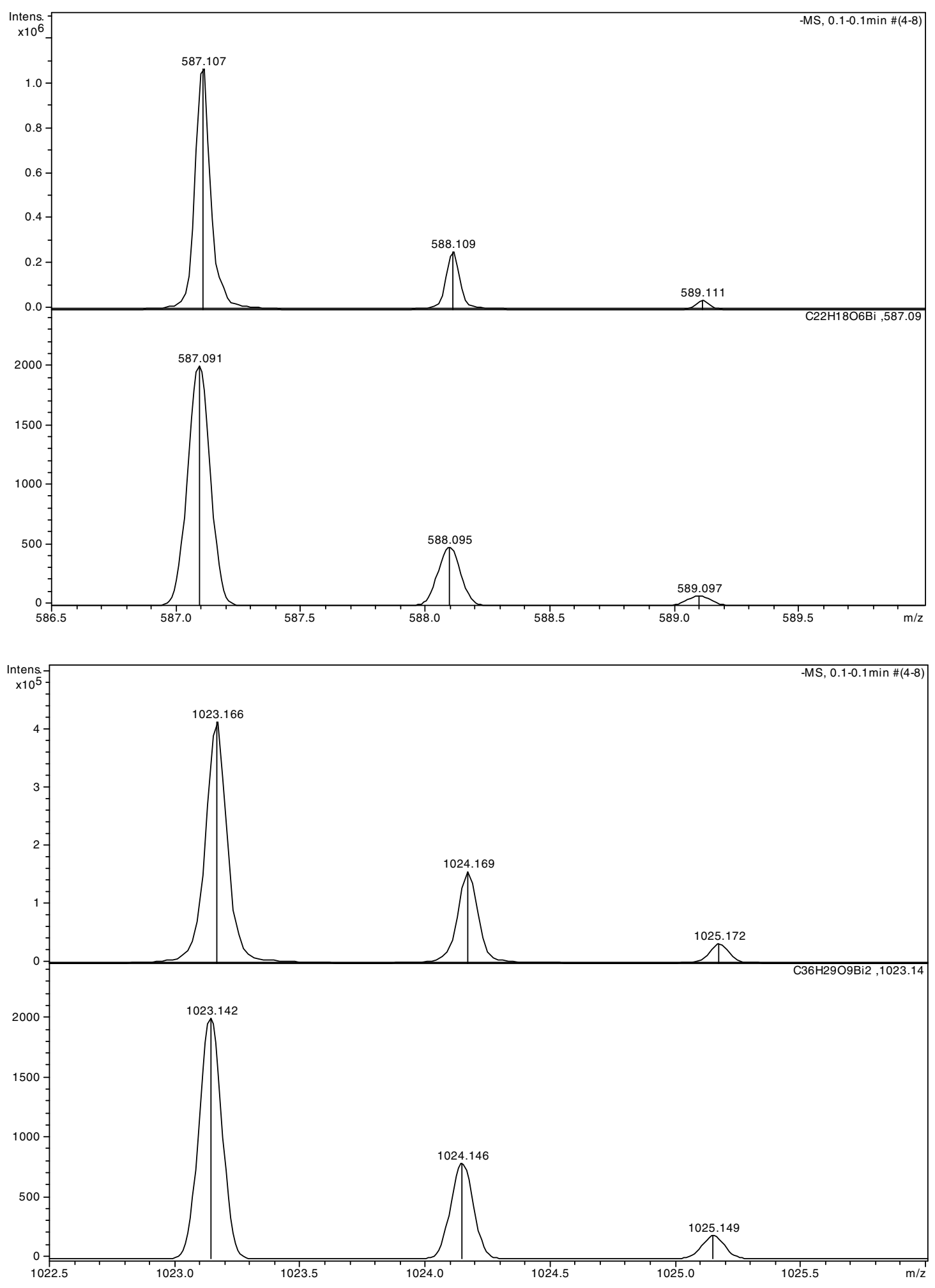

Figure S3. Observed and calculated isotope distributions for (a) $\left[\mathrm{BiPh}\left(\mathrm{Hsal}^{4 \mathrm{Me}}\right)\left(\mathrm{sal}^{4 \mathrm{Me}}\right)\right]^{-}$and (b) $\left[\mathrm{Bi}_{2} \mathrm{Ph}_{2}\left(\mathrm{Hsal}^{4 \mathrm{Me}}\right)\left(\mathrm{sal}^{4 \mathrm{Me}}\right)_{2}\right]^{-}$in ESI-MS. 
(b)

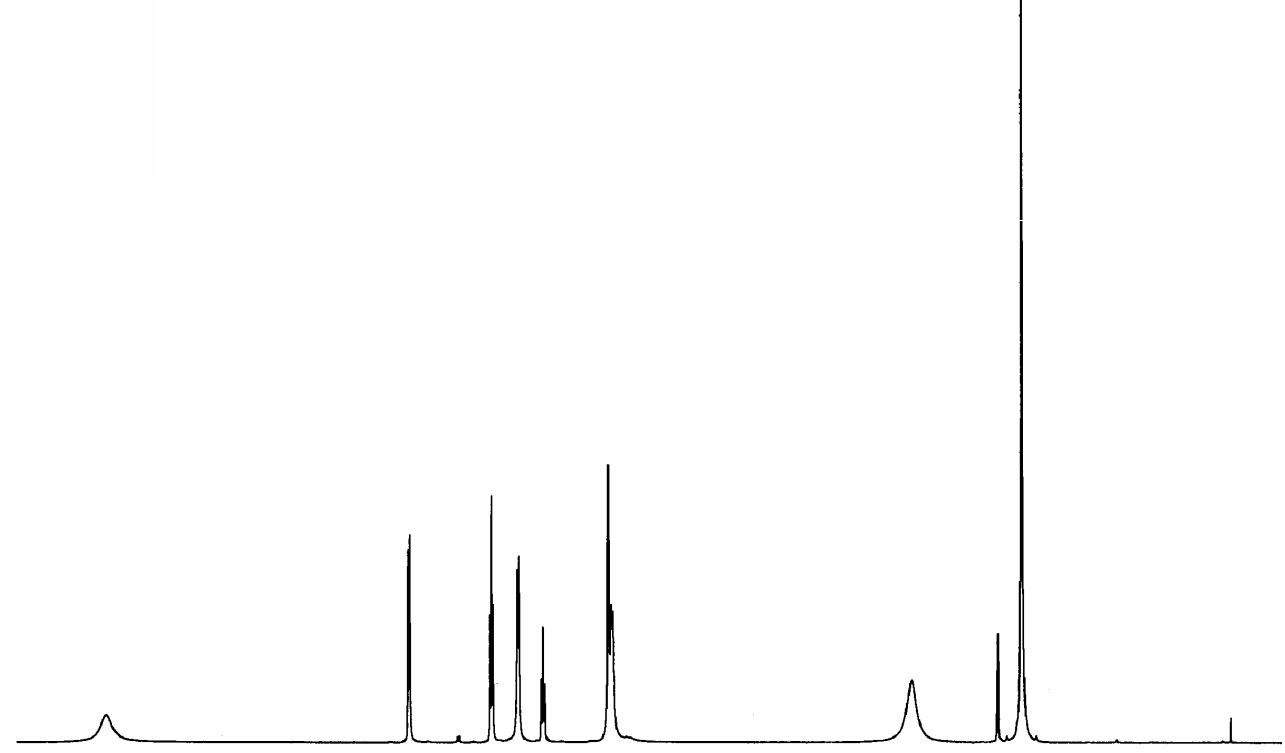

(a)

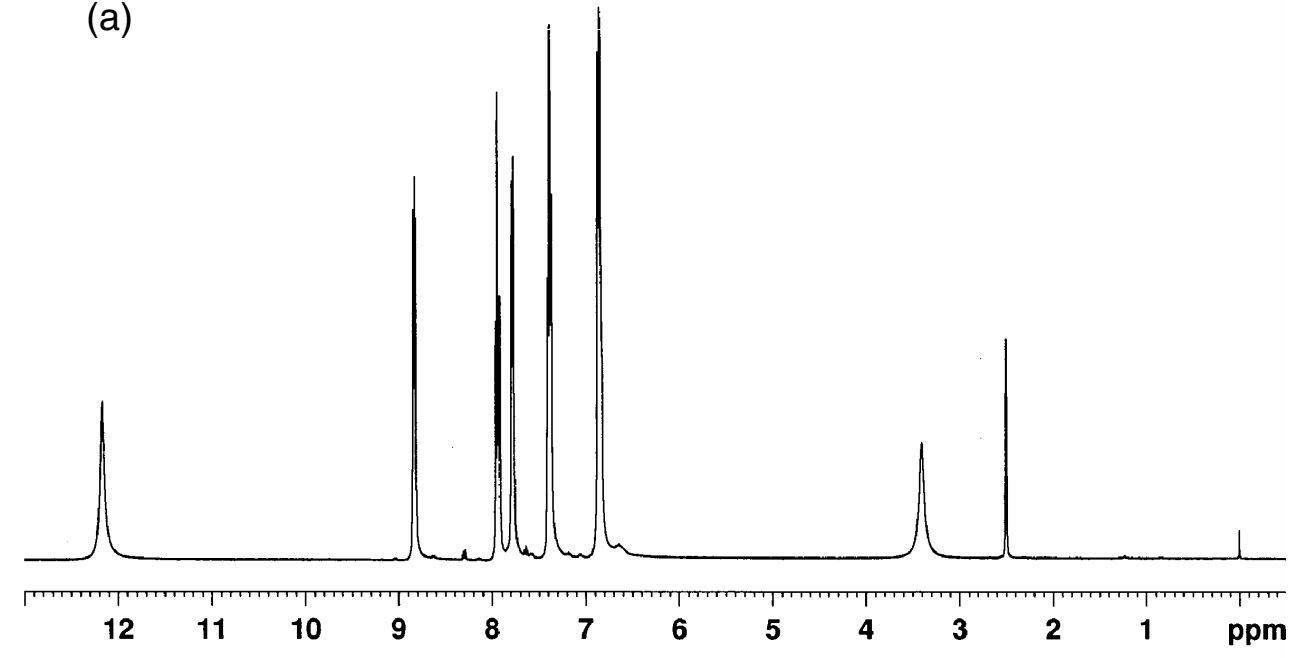

Figure S4. ${ }^{1} \mathrm{H}$ NMR spectra of (a) $\mathbf{1 a}$ and (b) $\mathbf{1 b}$ in $\mathrm{d}_{6}$-dmso $(400 \mathrm{MHz})$. 
(a)
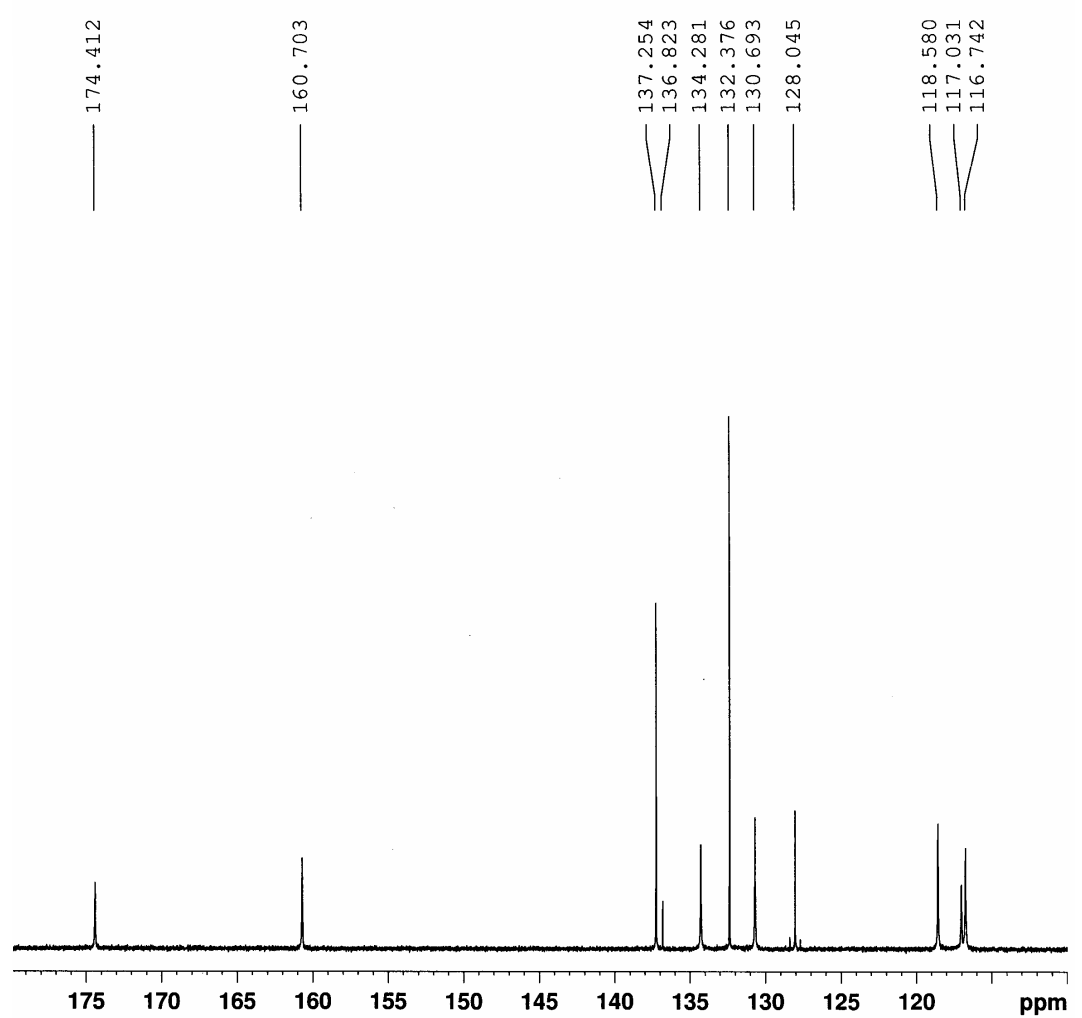

(b)

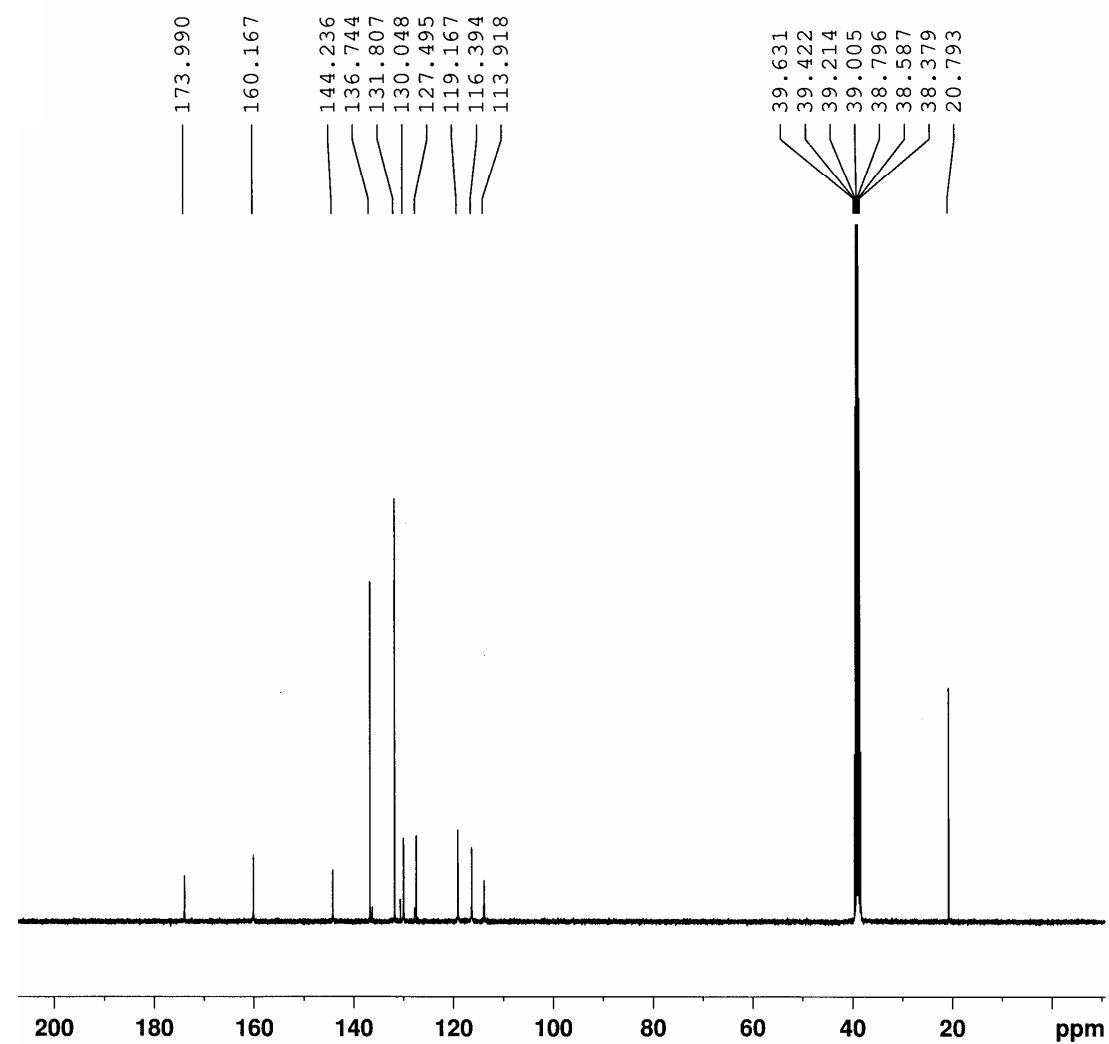

Figure S5. ${ }^{13} \mathrm{C}\left\{{ }^{1} \mathrm{H}\right\}$ spectra of (a) $\mathbf{1 a}$ and (b) $\mathbf{1 b}$ in $\mathrm{d}_{6}$-dmso $(400 \mathrm{MHz})$. 
(a)

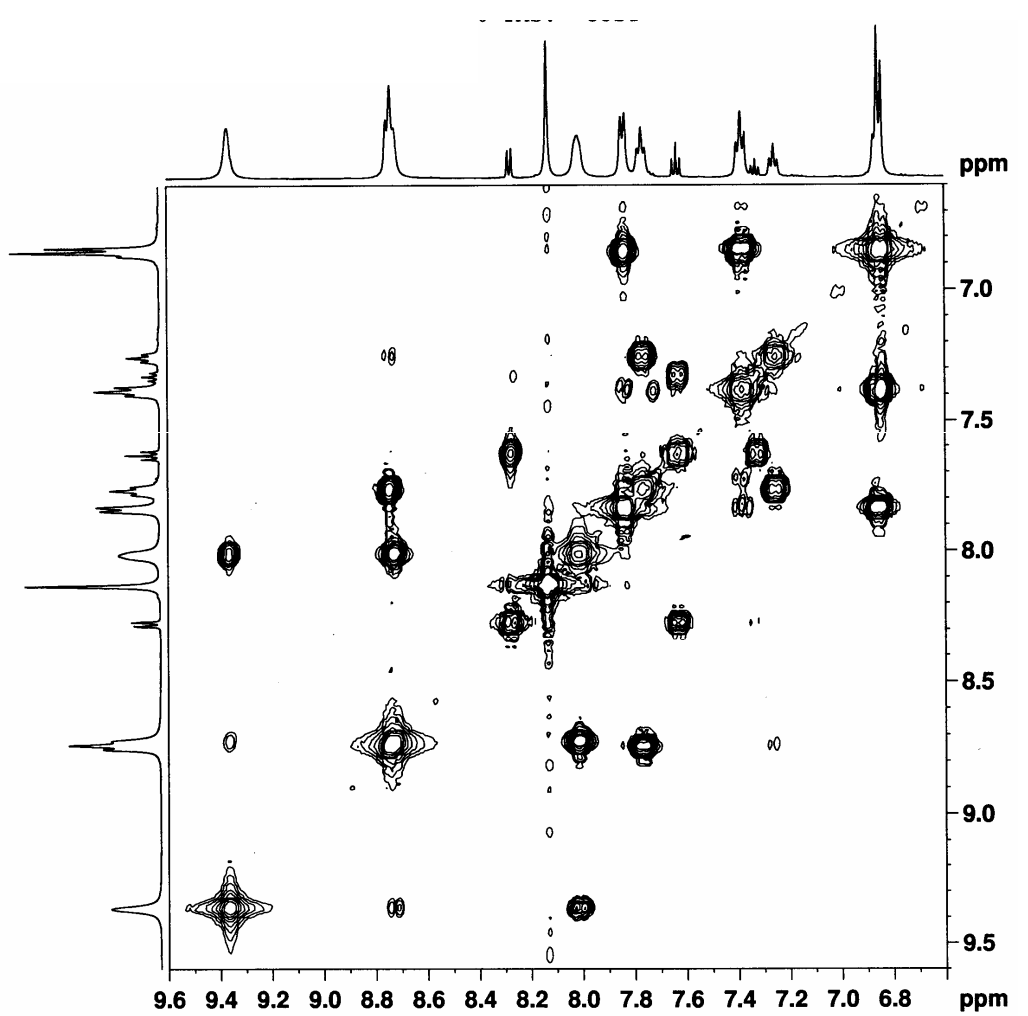

(b)

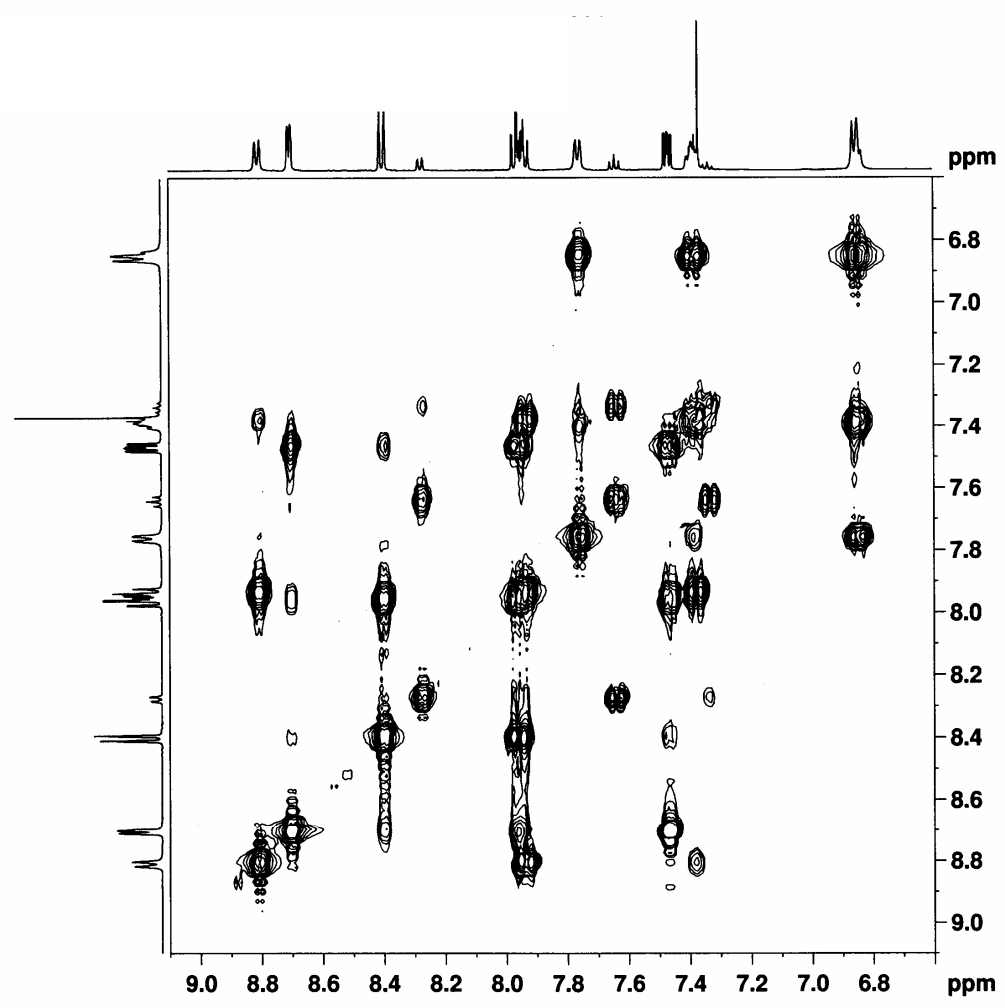

Figure S6. ${ }^{1} \mathrm{H}-{ }^{1} \mathrm{H}$ COSY NMR spectra of (a) $2 \mathbf{a}$ and (b) $2 \mathbf{c}$ in $\mathrm{d}_{6}$-dmso (500 MHz). Low intensity signals at $\sim 8.3 \mathrm{ppm}$, and the pair of triplets at $\sim 7.6 \mathrm{ppm}$ and $\sim 7.3$ may be attributed to the presence of traces of diphenylbismuth(salicylate) species in solutions. 

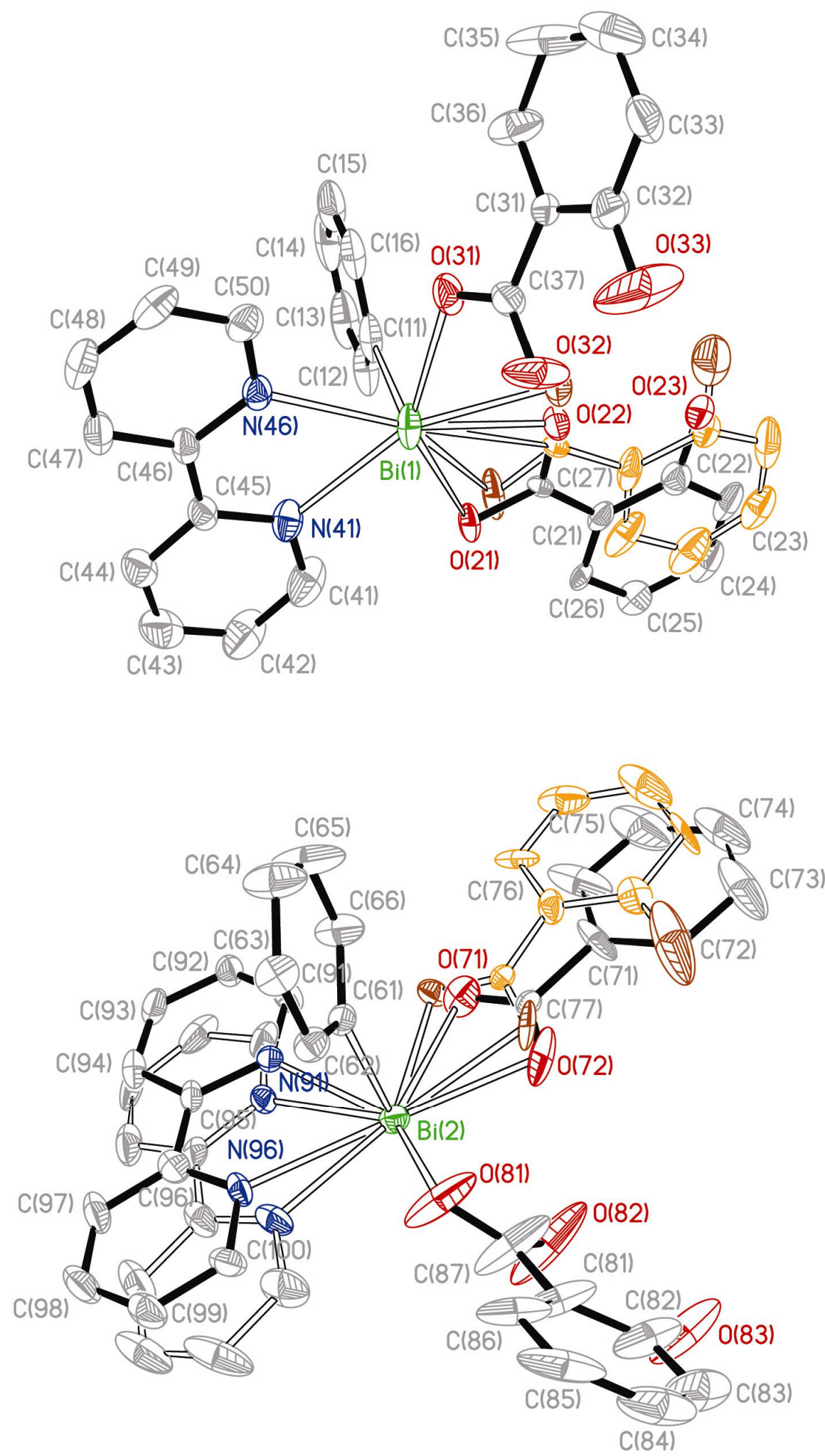

Figure S7. Coordination environment of $\mathrm{Bi}(\mathrm{III})$ in the two molecules of the asymmetric unit of $\mathbf{2 c}$. 
Table S1. Selected geometric parameters for $\mathbf{2 c}$.

$\begin{array}{lc} & \text { Bond distances }(\AA) \\ \mathrm{Bi}(1)-\mathrm{C}(11) & 2.250(3) \\ \mathrm{Bi}(1)-\mathrm{O}(31) & 2.330(4) \\ \mathrm{Bi}(1)-\mathrm{N}(46) & 2.521(2) \\ \mathrm{Bi}(1)-\mathrm{O}(22) & 2.533(7) \\ \mathrm{Bi}(1)-\mathrm{O}(21) & 2.538(7) \\ \mathrm{Bi}(1)-\mathrm{O}(21 \mathrm{~A}) & 2.524(7) \\ \mathrm{Bi}(1)-\mathrm{O}(22 \mathrm{~A}) & 2.531(7) \\ \mathrm{Bi}(1)-\mathrm{N}(41) & 2.546(3) \\ \mathrm{Bi}(1)-\mathrm{C}(27 \mathrm{~A}) & 2.854(11) \\ \mathrm{Bi}(2)-\mathrm{C}(61) & 2.251(2) \\ \mathrm{Bi}(2)-\mathrm{O}(81) & 2.316(4) \\ \mathrm{Bi}(2)-\mathrm{N}(96 \mathrm{~A}) & 2.467(5) \\ \mathrm{Bi}(2)-\mathrm{N}(91 \mathrm{~A}) & 2.483(4) \\ \mathrm{Bi}(2)-\mathrm{O}(72) & 2.491(7) \\ \mathrm{Bi}(2)-\mathrm{O}(71) & 2.495(7) \\ \mathrm{Bi}(2)-\mathrm{O}(71 \mathrm{~A}) & 2.496(7) \\ \mathrm{Bi}(2)-\mathrm{O}(72 \mathrm{~A}) & 2.510(7) \\ \operatorname{Bi}(2)-\mathrm{N}(96) & 2.615(5) \\ \mathrm{Bi}(2)-\mathrm{N}(91) & 2.649(5) \\ \operatorname{Bi}(2)-\mathrm{C}(77) & 2.816(10)\end{array}$

Bond angles (deg.)

$\begin{array}{ll}\mathrm{C}(11)-\mathrm{Bi}(1)-\mathrm{O}(31) & 82.32(14) \\ \mathrm{C}(11)-\mathrm{Bi}(1)-\mathrm{N}(46) & 84.89(12) \\ \mathrm{O}(31)-\mathrm{Bi}(1)-\mathrm{N}(46) & 82.61(12) \\ \mathrm{C}(11)-\mathrm{Bi}(1)-\mathrm{O}(22) & 86.7(3) \\ \mathrm{O}(31)-\mathrm{Bi}(1)-\mathrm{O}(22) & 86.8(2) \\ \mathrm{N}(46)-\mathrm{Bi}(1)-\mathrm{O}(22) & 167.2(3) \\ \mathrm{C}(11)-\mathrm{Bi}(1)-\mathrm{O}(21) & 89.0(3) \\ \mathrm{O}(31)-\mathrm{Bi}(1)-\mathrm{O}(21) & 138.2(2) \\ \mathrm{N}(46)-\mathrm{Bi}(1)-\mathrm{O}(21) & 137.4(2) \\ \mathrm{O}(22)-\mathrm{Bi}(1)-\mathrm{O}(21) & 51.9(3) \\ \mathrm{C}(11)-\mathrm{Bi}(1)-\mathrm{N}(41) & 85.64(13) \\ \mathrm{O}(31)-\mathrm{Bi}(1)-\mathrm{N}(41) & 146.04(12) \\ \mathrm{N}(46)-\mathrm{Bi}(1)-\mathrm{N}(41) & 64.72(10) \\ \mathrm{O}(22)-\mathrm{Bi}(1)-\mathrm{N}(41) & 124.2(2) \\ \mathrm{O}(21)-\mathrm{Bi}(1)-\mathrm{N}(41) & 72.8(2) \\ \mathrm{C}(61)-\mathrm{Bi}(2)-\mathrm{O}(81) & 83.93(14) \\ \mathrm{C}(61)-\mathrm{Bi}(2)-\mathrm{O}(72) & 85.6(4) \\ \mathrm{O}(81)-\mathrm{Bi}(2)-\mathrm{O}(72) & 77.0(3) \\ \mathrm{C}(61)-\mathrm{Bi}(2)-\mathrm{O}(71) & 86.2(5) \\ \mathrm{O}(81)-\mathrm{Bi}(2)-\mathrm{O}(71) & 129.5(4) \\ \mathrm{O}(72)-\mathrm{Bi}(2)-\mathrm{O}(71) & 52.9(4) \\ \mathrm{C}(61)-\mathrm{Bi}(2)-\mathrm{N}(96) & 83.27(18) \\ \mathrm{O}(81)-\mathrm{Bi}(2)-\mathrm{N}(96) & 87.7(2) \\ \mathrm{O}(72)-\mathrm{Bi}(2)-\mathrm{N}(96) & 161.9(4) \\ \mathrm{O}(71)-\mathrm{Bi}(2)-\mathrm{N}(96) & 139.9(3) \\ \mathrm{C}(61)-\mathrm{Bi}(2)-\mathrm{N}(91) & 82.49(18) \\ \mathrm{O}(81)-\mathrm{Bi}(2)-\mathrm{N}(91) & 148.2(2) \\ \mathrm{O}(72)-\mathrm{Bi}(2)-\mathrm{N}(91) & 130.1(3) \\ \mathrm{O}(71)-\mathrm{Bi}(2)-\mathrm{N}(91) & 78.0(3) \\ \mathrm{N}(96)-\mathrm{Bi}(2)-\mathrm{N}(91) & 62.32(19) \\ & \\ & \\ & \end{array}$



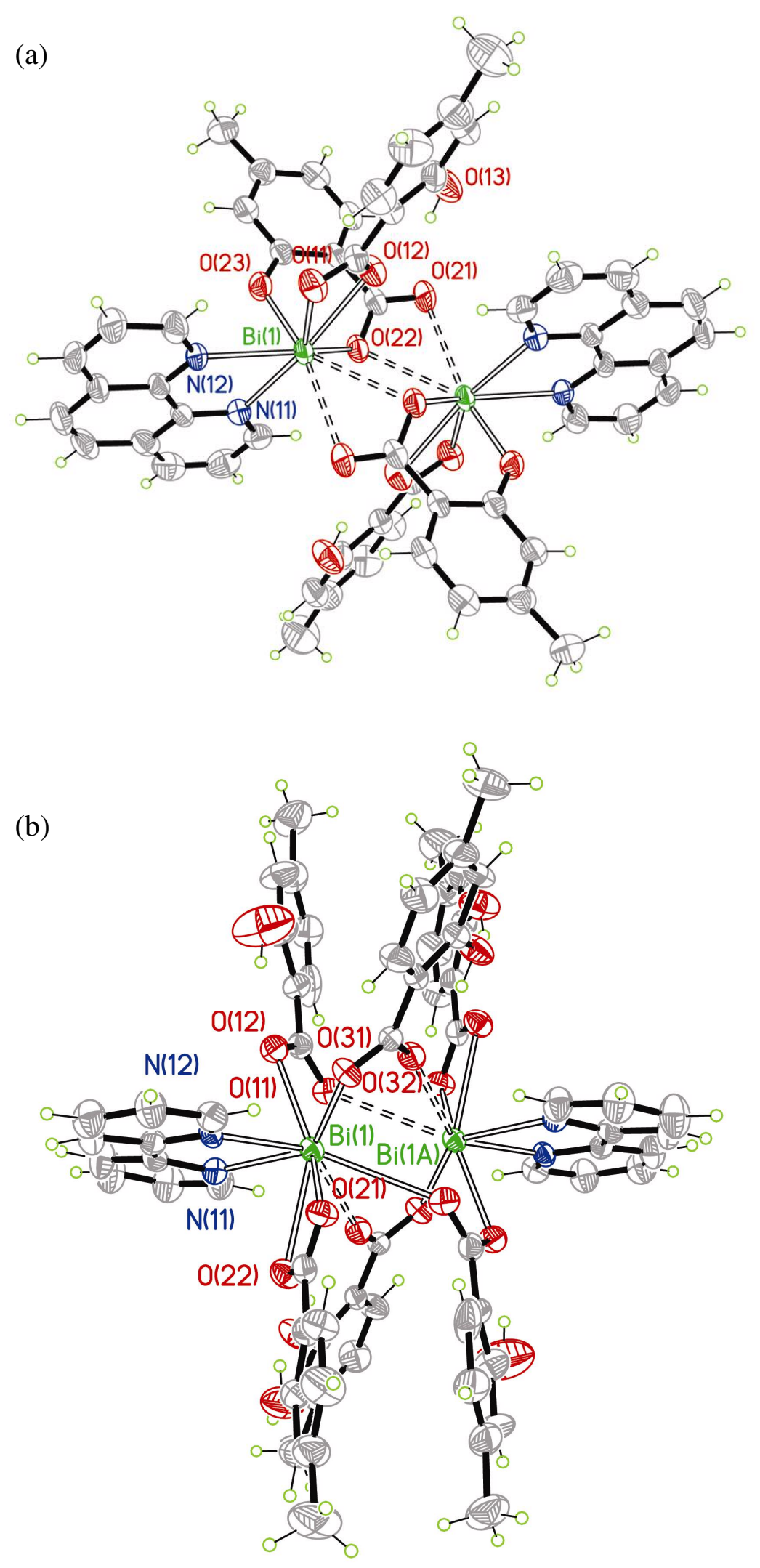

Figure S8. Coordination environment of $\mathrm{Bi}(\mathrm{III})$ in (a) $[3]_{2} \cdot 2 \mathrm{Me}_{2} \mathrm{CO}$ and (b) $[4]_{2} \cdot 4 \mathrm{Me}_{2} \mathrm{CO}$. 


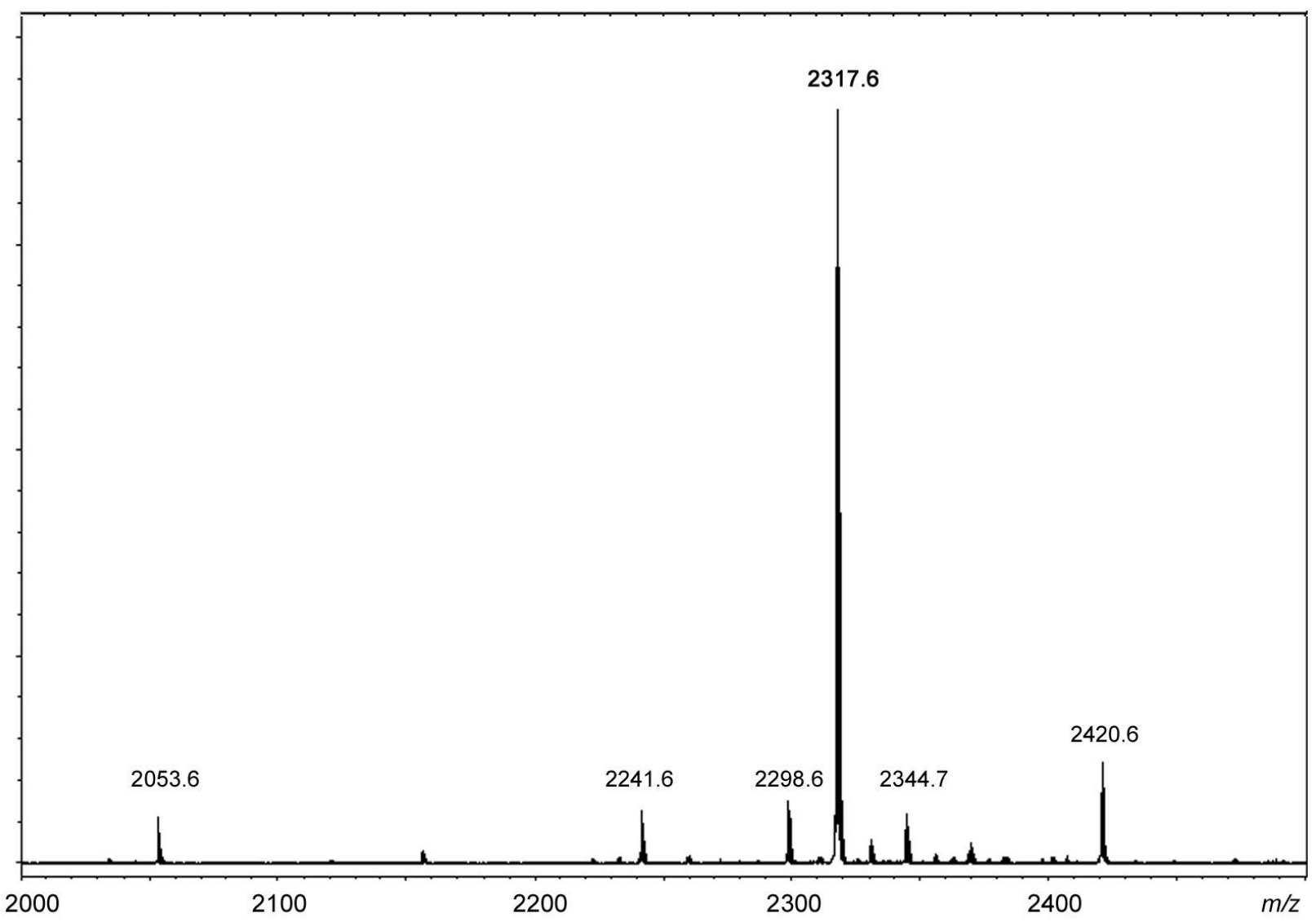

Figure S9. The negative-ion ESI mass spectrum of the product of the reaction of $\mathrm{BiPh}_{3}$ and $\mathrm{H}_{2} \mathrm{sal}^{4 \mathrm{Me}}$ in reagent-grade acetone 

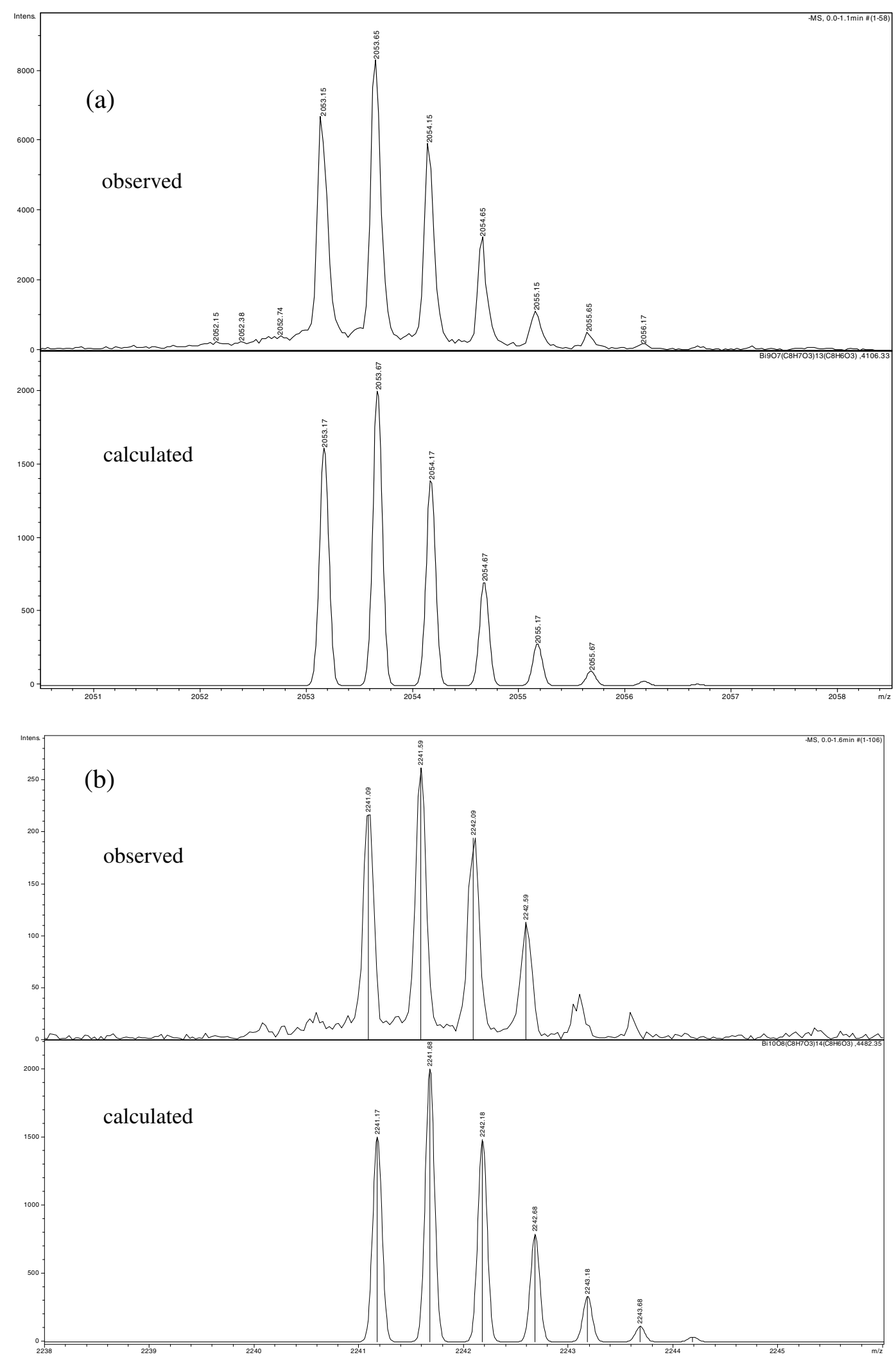

Figure S10. Comparison of experimental and calculated negative-ion ESI mass spectral isotope patterns for $\left[\mathrm{Bi}_{9} \mathrm{O}_{7}\left(\mathrm{Hsal}^{4 \mathrm{Me}}\right)_{13}(\mathrm{sal})\right]^{2-}(\mathbf{a})$ and $\left[\mathrm{Bi}_{10} \mathrm{O}_{8}\left(\mathrm{Hsal}^{4 \mathrm{Me}}\right)_{14}\left(\mathrm{sal}^{4 \mathrm{Me}}\right)\right]^{2-}(\mathbf{b})$. 

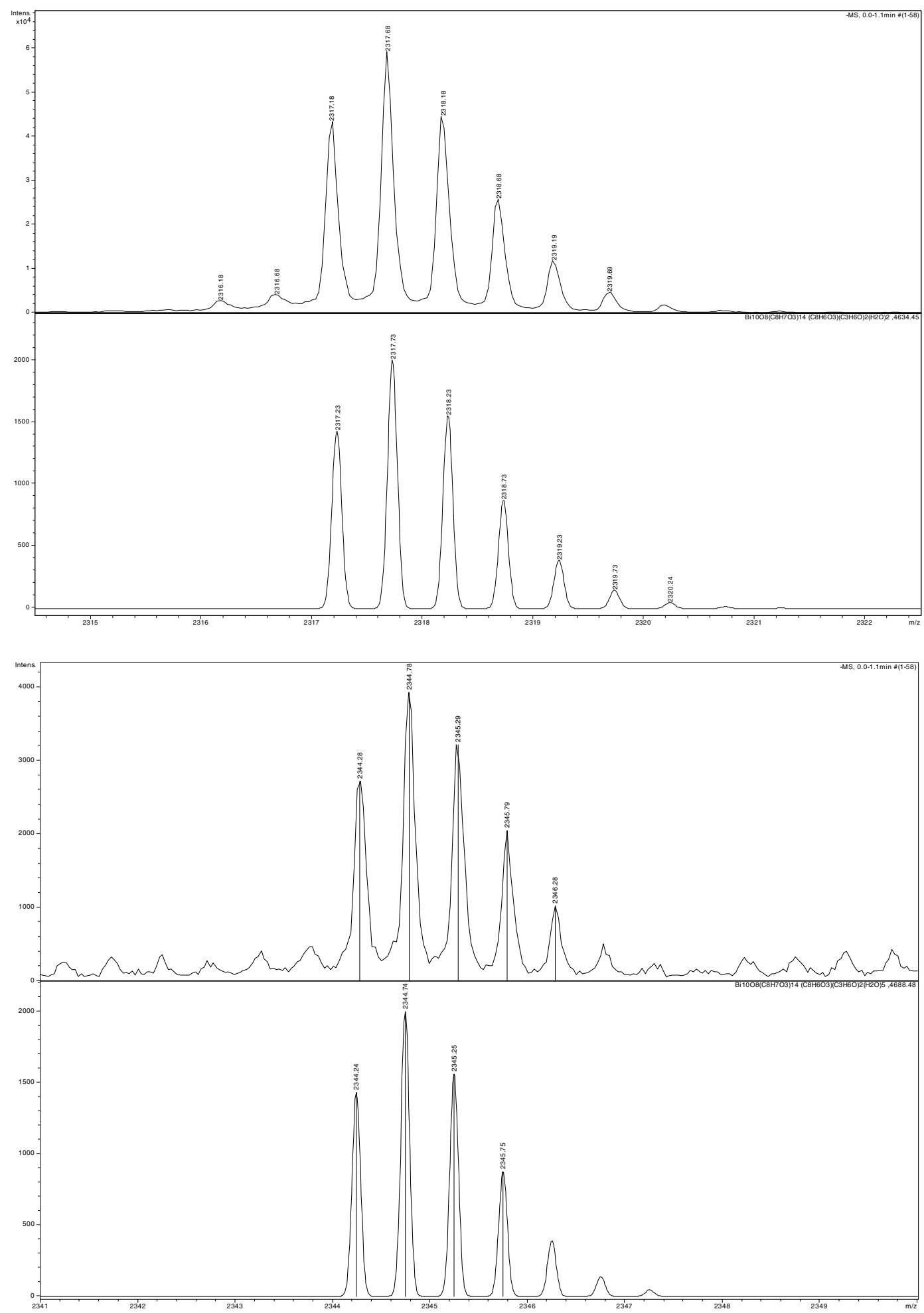

Figure S11. Comparison of experimental and calculated negative-ion ESI mass spectral isotope patterns for $\left[\mathrm{Bi}_{10} \mathrm{O}_{8}\left(\mathrm{Hsal}^{4 \mathrm{Me}}\right)_{14}\left(\mathrm{sal}^{4 \mathrm{Me}}\right)\left(\mathrm{Me}_{2} \mathrm{CO}\right)_{2}\left(\mathrm{H}_{2} \mathrm{O}\right)_{2}\right]^{2-}(\mathbf{a})$ and $\left[\mathrm{Bi}_{10} \mathrm{O}_{8}\left(\mathrm{Hsal}^{4 \mathrm{Me}^{2}}\right)_{14}\left(\mathrm{sal}^{4 \mathrm{Me}}\right)\left(\mathrm{Me}_{2} \mathrm{CO}\right)_{2}\left(\mathrm{H}_{2} \mathrm{O}\right)_{5}\right]^{2-}(\mathbf{b})$. 
Table S2. ATR FT-IR $\left(\mathrm{ZnSe}, \mathrm{cm}^{-1}\right)$ spectroscopic results s for complexes $\mathbf{1 a}, \mathbf{1 b}, \mathbf{2 a} \mathbf{- 2 d} \mathbf{2}$.

\begin{tabular}{|c|c|}
\hline Compound 1a & $\begin{array}{l}1620,1586,1518,1482,1465,1437,1400,1376,1309,1253,1224,1159,1142, \\
1055,1031,997,879,872,825,817,809,763,752,731,702,668,647,560, \\
534,526\end{array}$ \\
\hline Compound $\mathbf{1 b}$ & $\begin{array}{l}1629,1577,1560,1491,1415,1341,1305,1251,1166,1156,1056,999,948, \\
861,848,782,734,698,630,587,532\end{array}$ \\
\hline Compound 2a & $\begin{array}{l}1622,1595,1566,1524,1482,1458,1374,1317,1306,1254,1237,1189,1159, \\
1142,1100,1055,1031,997,929,871,862,817,752,731,725,703,694,666, \\
637,602,573,560,526,515\end{array}$ \\
\hline Compound $\mathbf{2 b}$ & $\begin{array}{l}1632,1582,1561,1496,1474,1422,1412,1361,1342,1306,1255,1223,1164, \\
1131,1099,1055,1010,998,948,834,825,790,781,722,702,690,639,621, \\
588,554,529,507 .\end{array}$ \\
\hline Compound 2c & $\begin{array}{l}1619,1593,1563,1538,1484,1474,1455,1446,1437,1379,1365,1306,1251, \\
1156,1140,1062,1029,1010,996,867,861,815,756,740,731,704,696,665, \\
643,625,556,534\end{array}$ \\
\hline Compound 2d & $\begin{array}{l}1629,1577,1552,1491,1409,1370,1332,1305,1250,1219,1163,1098,1059, \\
1011,994,948,844,826,781,763,732,695,618,587,540,530,507 .\end{array}$ \\
\hline Compound $\mathbf{5}$ & $\begin{array}{l}1636,1583,1521,1496,1429,1418,1390,1339,1308,1256,1225,1165,1149, \\
1105,1027,950,866,849,833,787,774,736,725,720,700,644,627,617, \\
598,577,558,532,524,514 .\end{array}$ \\
\hline
\end{tabular}

